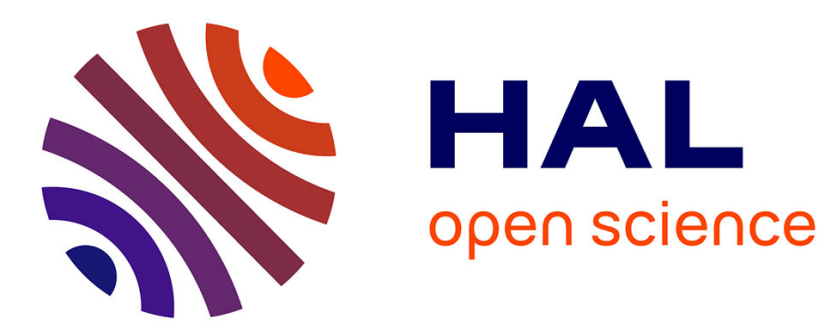

\title{
Efficient Exact Arithmetic over Constructive Reals
}

\author{
Yong Li, Jun-Hai Yong
}

\section{To cite this version:}

Yong Li, Jun-Hai Yong. Efficient Exact Arithmetic over Constructive Reals. The 4th Annual Conference on Theory and Applications of Models of Computation, May 2007, Shanghai, China. inria00517598

\section{HAL Id: inria-00517598 \\ https://hal.inria.fr/inria-00517598}

Submitted on 15 Sep 2010

HAL is a multi-disciplinary open access archive for the deposit and dissemination of scientific research documents, whether they are published or not. The documents may come from teaching and research institutions in France or abroad, or from public or private research centers.
L'archive ouverte pluridisciplinaire HAL, est destinée au dépôt et à la diffusion de documents scientifiques de niveau recherche, publiés ou non, émanant des établissements d'enseignement et de recherche français ou étrangers, des laboratoires publics ou privés. 


\title{
Efficient Exact Arithmetic over Constructive Reals
}

\author{
Yong Li and Jun-Hai Yong \\ School of Software, Tsinghua University, Beijing 100084, P.R. China \\ exactreal@yahoo.com.cn
}

\begin{abstract}
We describe a computing method of the computable (or constructive) real numbers based on analysis of expressions. This method take precision estimate into account in order to get a better algorithm than Ménissier-Morain's method, which is also based on the representation of constructive reals. We solve two problems which appear in exact real arithmetic based on the representation of constructive reals. First, by balancing every item's precision in the expression, we can avoid unnecessary precision growth. Second, by distributing different weights to different operations, we can make sure that complex operations do not waste much time when to compute the whole expression. In these ways, we finally get a more efficient and proper method than prior implementations.
\end{abstract}

\section{Introduction}

The goal of exact arithmetic is to generate correct answers to numeric problems, within some user-specified error. Generally speaking, floating-point numbers are widely used to represent real numbers in computer systems, although the limitations are quit obvious. For instance, the real numbers cannot be arbitrarily large or small, and the rounding errors can lead to inaccurate or completely wrong results. Some examples can be found in 112. In order to solve these limitations, many methods have been proposed, one of which is exact arithmetic that has attracted more and more attentions in recent years.

Several methods have been developed for exact arithmetic which can supply an approximation of the exact result that meets the user's any requirement of precise. V. Ménissier-Morain in [3] gives a brief introduction of these methods. However, only two of them are practical and proved completely correct. One is linear fractional transformation (LFT) which is mostly based on continued fractions. In 1976, Gosper first introduced this mthod to calculate the operations of real numbers [4. In 1989, Vuillemin implemented the arithmetic using Lisp and improved this arithmetic in many ways [5. In 2002, Edalat and Heckmann developed this arithmetic to LFT Framework [1] and they also supply an implementation in Haskell and C, which is named IC-Reals. The other one, constructive reals, is based on Cauchy Sequences, a theory that is well established by Bishop [6. It can also be considered as a foundation of computable analysis. Boehm and Lee first made use of this theory for the purpose of exact arithmetic [78] and Boehm finished this algorithm using java [9]. Gowland and Lester in 
[10]11 showed us the correctness of an implementation based on fast binary Cauchy sequence, which makes the base equal to 2 . And they have described a Haskell implementation in 10. In addition, V. Ménissier-Morain in $[3$ proved a similar algorithm whose base can be 2 or other integers which is bigger than 2 . Briggs implemented this paper's algorithm using Python, $\mathrm{C}++, \mathrm{C}$ respectively [12. Of course, there are also many other implementations and you can get more information about exact real arithmetic in this survey [13. But most of them are deficient in the proof or integrality of algorithms.

Actually, all these methods can be converted into interval representations. But different methods have different algorithms to calculate the operation concerning real numbers. In that case their performances are also different. In terms of basic operations, like addition, minus, multiplication and division, the second method may be more efficient, which can be found in this competence result in 2000 14. However, LFT approach also has some merits that it can easily handle irrational numbers 15|16, that Edalat argues its transcendental functions may more efficient, and that under special circumstances continued fractions can solve many problems which common representations is hard to handle. In this paper, our interest focus on the amelioration of the second methods, that is constructive reals. And we give new algorithms that solve two problems which can affect the implementation's performance. First, by balancing every item's precision, we can avoid unnecessary precision growth which is the first problem that has attracted many attentions in 17/18. Second, by distributing different weights to different operations, we can make sure that complex operations do not waste most time when to compute the whole expression which is the second problem that we proposed.

The remaining part of the paper is arranged as follows. Related definitions and algorithms about Cauchy sequences are briefly introduced in section 2. Problems of present implementations based on Cauchy sequences are shown in section 3 . A new simple algorithm about addition, which considers balancing every item's precision, is proved in section 4 . And a new algorithm about calculating the expression that takes every operation's complexity into consideration will be given in section5. The experimental result and discussion are set in section 6 .

\section{Basic Definitions and Algorithms}

We present some basic definitions and algorithms of computable real numbers, which derived from Boehm, V. Ménissier-Morain, and Gowland's work in [39]10]. First, we will show two definitions about the representation of real numbers. Second, we will give two simple algorithms about addition and multiplication that will help us understand the problem.

Definition 1. (Effective Cauchy sequences). A computable real number $x$ is represented as an Effective Cauchy sequence, if there is an infinite computable sequence of rational numbers $\left\{\frac{n_{0}}{d_{0}}, \frac{n_{1}}{d_{1}}, \ldots, \frac{n_{p}}{d_{p}}, \ldots,\right\}$, with $d_{i}>0$, and a modulus 
of convergence function $e: N \rightarrow N$ which is recursive, such that $\forall p \in \mathbf{N}: k \geq$ $e(p)$ implies $\left|x-\frac{n_{k}}{d_{k}}\right|<2^{-p}$.

In this definition, we use a sequence of rational numbers to represent the real number. When we need to get a number which meets the precision requirement, we can take a proper rational number from the sequence. In fact, we can get an interval which is small enough to meet the error range and includes the true value of the real number that is $x \in\left(\frac{n_{k}}{d_{k}}-2^{-p}, \frac{n_{k}}{d_{k}}+2^{-p}\right)$. But if we only want to use integer arithmetic to finish this kind of exact real arithmetic, we should do some changes.

Definition 2. (Fast Binary Cauchy Sequence) A computable real number $x$ is represented as a Fast Binary Cauchy Sequence if there is an infinite computable sequence of integers $\left\{n_{0}, n_{1}, \ldots, n_{p}, \ldots\right\}$, such that $\left|x-2^{-p} n_{p}\right|<2^{-p}$.

This definition makes a little change with respect to Definition 1 . We can use a sequence of integers to represent real number, which makes implementation only use integer arithmetic and is not confined by floating-point numbers' defects. Some implementations using floating point, such as IRRAM 19, which is based on real-RAMs, have faster speed. Although some real-RAMs can be realized approximately by floating point computations, real-RAMs cannot be realized by physical machines, they are unrealistic [20. But the way of construct reals is completely accepted in that it only uses integer arithmetic. So in section 5 we do not compare time with IRRAM which use floating-point arithmetic in implementation. In this definition, if the error range is $2^{-p}$, we can use $n_{p}$ to represent the real number $x$, which meets the requirement of $x \in\left(2^{-p} n_{p}-2^{-p}, 2^{-p} n_{p}+2^{-p}\right)$. In the rest of the article, we use $x[p]$ to represent $n_{p}$.

Also, there are other similar definitions, in 3 , they use the qualification of $\left|x-\frac{n_{k}}{d_{k}}\right|<B^{-p}$ where $B \geq 2$ is some fixed integer, instead of $\left|x-\frac{n_{k}}{d_{k}}\right|<2^{-p}$ in Definition 1. Obviously, modulating $B$ can control the interval's and the error range. For example, if $B$ is 10 , we can get a sequence of error range: $10^{-1}, 10^{-2}$, $\ldots, 10^{-p}, \ldots$ If $B$ is 2 , we can get a sequence of error range: $2^{-1}, 2^{-2}, \ldots$, $2^{-p}, \ldots$ which is more flexible than $B$ is 10 and can easily meet more error requirements. In this paper, we only discuss our implementation based on $B=2$ and these circumstances that $B>2$ can be handled in the same thought.

Now we simply give some algorithms concerning addition and multiplication which is widely used in 3 910] and is showed in [10]. These algorithms can help us to illustrate our algorithms. Their proofs can be found in 10. We suppose that $x, x_{1}, x_{2}$ are real numbers and the required error range of $x$ is $2^{-p}$ which means we need to get $x[p]$.

Algorithm 1. (Addition of real numbers) Computing $x=x_{1}+x_{2}$, we have $x[p]=\operatorname{round}\left(\left(x_{1}[p+2]+x_{2}[p+2]\right) / 4\right)$.

Algorithm 2. (Multiplication of real numbers) Computing $x=x_{1} * x_{2}$, we have $x[p]=\operatorname{round}\left(2^{-\left(p+s_{1}+s_{2}\right)}\left(n_{1} n_{2}\right)\right)$ where $s_{1}=\left\lfloor\log _{2}\left(\left|x_{1}[0]\right|+2\right)\right\rfloor+3$ and $s_{2}=\left\lfloor\log _{2}\left(\left|x_{2}[0]\right|+2\right)\right\rfloor+3$. 


\section{Problems}

Several authors have implemented the algorithms that are based on constructive reals. We can divide them into two kinds. One is to use Functional language which is easy and natural to implement exact real arithmetic in that we can regard a real number as a function and the expression can be evaluated automatically. However, this way is very slow and impractical in many applications. The other is to use DAG (directed acyclic graph) to represent the expression about operations between exact real numbers. For example, Figure 1 shows an expression based on DAG. This way can be finished by using $\mathrm{C}$ language, which can get much better performance and also is easier to use in other software. But both of them suffer from several flaws.

In the first problem, with the increase of operations' number, the original algorithms fail to use proper precise of every real number. For example, we want to compute $x=x_{1}+x_{2}+x_{3}+x_{4}$ according to Algorithm 1. Its DAG is figure 1 , and when we want to get $x[p]$, we need get $x_{4}[p+2], x_{3}[p+4]$, $x_{2}[p+6], x_{1}[p+8]$ respectively. Obviously, when the items' number is very large we may need some items that have extremely small precision. However, by scrutinizing this case carefully, we find it is not reasonable. If we consider it from a prospect of expression we find if we want to get an approximation of $x$ up to the precision $\epsilon$, we only need every item' approximation up to $\epsilon / 4$. In this case, we should balance every item's precision to get a more proper means to compute the expression. This problem has been proposed in [17]18, which occurs in all exact real arithmetic based on Type2 theory [20. In [18, the author proposed that each item's precision should be balanced to solve this problem. But in special representation how to solve it is also a question. In this paper we give the methods about constructive reals and give a proof in the follow section.

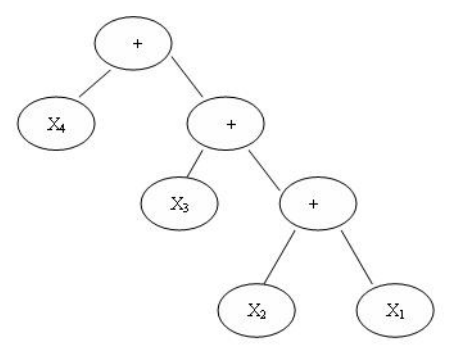

Fig. 1. A DAG for the expression $x_{1}+x_{2}+x_{3}+x_{4}$

In the second problem, the original algorithm fails to take the complexity of each operation into consideration. Obviously, computing one multiplication takes more time than computing an addition when we need them to get the same precision which can be found in Algorithm 1 and Algorithm 2. The original algorithms 
are not concerned about making the precision of operation of multiplication as large as possible. For instance, when we compute $x=x_{1}+x_{2} * x_{3}$, in order to get $x[p]$, a choice is to get $x_{1}[p+2]$ and $\left(x_{2} * x_{3}\right)[p+2]$ which is an original algorithm's way. However, in terms of expression, we have many other ways. For example, when we want to get an approximation of $x$ up to the precision $\epsilon$, we also need $x_{1}$ 's approximation up to $\epsilon / 4$ and $\left(x_{2} * x_{3}\right)$ 's approximation up to $3 \epsilon / 4$. In this case, the operation of addition will cost more time than the former and the operation of multiplication will cost little time than the former. But we think it is totally worthy because the multiplication is more complex.

In conclusion, original implementations do not consider the difference between exact real arithmetic and traditional computation. Traditional computation can use a common way to compute because they are not affected by error requirement. However, in exact arithmetic, distributing a proper precision to every item is very important which can make computation more effective.

\section{Addition Algorithm with Balanced Precision}

Addition and subtraction are very important in exact arithmetic in that summation plays an important role in science computing. And addition and subtraction's error analysis are also very easy to process. In this part we give an algorithm to compute an expression only including addition. Subtraction can be processed in a similar way in that it can be taken as a special addition.

Algorithm 3. Computing $x=x_{1}+x_{2}+\ldots+x_{n}$, where $x, x_{1}, x_{2}, \ldots, x_{n}$ are real numbers and the required of error range of $x$ is $2^{-p}$ which means we need to get $x[p]$, we have $x[p]=\operatorname{round}\left(\left(x_{1}[m]+x_{2}[m]+\ldots+x_{n}[m]\right) 2^{p-m}\right)$. where $m=\left\lceil\log _{2} n+p+1\right\rceil$.

Proof. We will prove that $\left|x-2^{-p} x[p]\right|=\left|x_{1}+x_{2}+\ldots+x_{n}-2^{-p} x[p]\right|<2^{-p}$ i.e.

$$
2^{-p}(x[p]-1)<x_{1}+x_{2}+\ldots+x_{n}<2^{-p}(x[p]+1)
$$

According to definition 2, we have

$$
\left|x-\left(x_{1}[m]+x_{2}[m]+\ldots+x_{n}[m]\right) 2^{-m}\right|, n 2^{-m}
$$

We make $m=\left\lceil\log _{2} n+p+1\right\rceil$ such that

$$
\frac{1}{2^{m}} \leq \frac{1}{n 2^{p+1}}<\frac{1}{2^{m-1}}
$$

According to (2) and (3), we get

$$
\begin{aligned}
& \left|x-\left(x_{1}[m]+x_{2}[m]+\ldots+x_{n}[m]\right) 2^{-m}\right|<n 2^{-m} \leq 2^{-(p+1)} \text { and } \\
& \left|x-\left(x_{1}[m]+x_{2}[m]+\ldots+x_{n}[m]\right) 2^{p-m} 2^{-p}\right|<2^{-(p+1)}
\end{aligned}
$$


Then according to round's property, we have

$$
\begin{aligned}
& \mid\left(x_{1}[m]+x_{2}[m]+\ldots+x_{n}[m]\right) 2^{p-m}-\operatorname{round}\left(\left(x_{1}[m]+x_{2}[m]+\ldots+x_{n}[m]\right)\right. \\
& \left.2^{p-m}\right) \mid \leq \frac{1}{2}
\end{aligned}
$$

By multiplying $2^{-p}$, Formula (5) can be changed into:

$$
\begin{aligned}
& \mid\left(x_{1}[m]+x_{2}[m]+\ldots+x_{n}[m]\right) 2^{-m}-\operatorname{round}\left(\left(x_{1}[m]+x_{2}[m]+\ldots+x_{n}[m]\right)\right. \\
& \left.\left.2^{p-m}\right) 2^{-p}\right) \mid \leq 2^{-(p+1)}
\end{aligned}
$$

Then we can use $\operatorname{round}\left(\left(x_{1}[m]+x_{2}[m]+\ldots+x_{n}[m]\right) 2^{p-m}\right)$ to replace $\left(x_{1}[m]+\right.$ $\left.x_{2}[m]+\ldots+x_{n}[m]\right) 2^{p-m}$ in Formula (4), we can get

$\left|x-\operatorname{round}\left(\left(x_{1}[m]+x_{2}[m]+\ldots+x_{n}[m]\right) 2^{p-m}\right) 2^{-p}\right|<2^{-(p+1)}+2^{-(p+1)}=2^{-p}$

i.e.

$$
\left|x-2^{-p} x[p]\right|=\left|x_{1}+x_{2}+\ldots+x_{n}-2^{-p} x[p]\right|<2^{-p}
$$

In this addition algorithm, we distribute the same precision to every item. For example, if we want to get $x[p]$ whose expression is shown in Figure 1, we only need get $x_{1}[p+3], x_{2}[p+3], x_{3}[p+3]$ and $x_{4}[p+3]$ respectively. When $\mathrm{n}$ is a very large number this way can evidently reduce many items' precision required. Compared with the original algorithm, this way is more proper in terms of expression.

\section{Algorithm with Precision Control}

In section 4, we have solved the first problem that exits in constructive reals' computation. In this section we will consider solving the second problem. From what have been discussed, we can see that different operations have different complexity. A sin's operation is more complex than addition operation when is computed to up to the same precision. Our solution is to distribute different weight to single computation cell. The weight can control the precision that operation needs. First, we introduce a definition of single computation cell.

Definition 3. (Single Computation Cell) A single computation cell is one of these cases: 1. A real number that interacts with other items only with addition or subtraction. 2. A result number that we get from operations except addition and subtraction and it interacts with other SCCs only with addition or subtraction.

For example, when we want to deal with $x_{1}+x_{2} * x_{3}+\sin x_{4}+x_{5} * x_{6} / x_{7}$, the number $x_{1}$, the result of $x_{2} * x_{3}$, the result of $\sin x_{4}$ and the result of $x_{5} * x_{6} / x_{7}$ are SCCs. We can simply put $x_{1}$ 's weight is $1, x_{2} * x_{3}$ is $4, \sin x_{4}$ is 16 and 
$x_{5} * x_{6} / x_{7}$ is 8 . The value of weight is based on the compute complexity of the SCC and is regulated to be the form of $2^{k}, k \in N$, which make our computation convenient. We can compute single SCC use its original algorithm, but compute the whole expression use our algorithm. Now we will show an algorithm that can used to compute these expressions.

Algorithm 4. If we want to compute $x=x_{1}+x_{2}+\ldots+x_{n}$ in order to meet some error range $2^{-p}$, where $x_{i}$ 's weight is $w_{i}$ and every $x_{i}$ is a $S C C, i=1,2, \ldots, n$. We have $x[p]=\operatorname{round}\left(x_{1}\left[m-\log _{2} w_{1}\right] w_{1} 2^{p-m}+x_{2}\left[m-\log _{2} w_{2}\right] w_{2} 2^{p-m}+\ldots+\right.$ $\left.x_{n}\left[m-\log _{2} w_{n}\right] w_{n} 2^{p-m}\right)$ with $m=\left\lceil-\log _{2}\left(\frac{2^{-(p+1)}}{w_{1}+w_{2}+\ldots+w_{n}}\right)\right\rceil$.

Proof. We will prove

$$
\left|x-2^{-p} x[p]\right|=\left|x_{1}+x_{2}+\ldots+x_{n}-2^{-p} x[p]\right|<2^{-p}
$$

We make $m=\left\lceil-\log _{2}\left(\frac{2^{-(p+1)}}{w_{1}+w_{2}+\ldots+w_{n}}\right)\right\rceil$ such that

$$
\left(w_{1}+w_{2}+\ldots+w_{n}\right) 2^{-m} \leq 2^{-(p+1)}
$$

According to definition 2, we have

$$
\begin{aligned}
& \mid x-\left(x_{1}\left[m-\log _{2} w_{1}\right] 2^{\log _{2} w_{1}-m}+x_{2}\left[m-\log _{2} w_{2}\right] 2^{\log _{2} w_{2}-m}+\ldots+x_{n}[m-\right. \\
& \left.\left.\log _{2} w_{n}\right] 2^{\log _{2} w_{n}-m}\right)|=| x-\left(x_{1}\left[m-\log _{2} w_{1}\right] w_{1} 2^{-m}+x_{2}\left[m-\log _{2} w_{2}\right] w_{2} 2^{-m}\right. \\
& \left.+\ldots+x_{n}\left[m-\log _{2} w_{n}\right] w_{n} 2^{-m}\right) \mid<\left(w_{1}+w_{2}+\ldots+w_{n}\right) 2^{-m} \leq 2^{-(p+1)}
\end{aligned}
$$

It is similar to the addition's proof above, we can get

$$
\begin{aligned}
& \mid\left(x_{1}\left[m-\log _{2} w_{1}\right] w_{1} 2^{-m}+x_{2}\left[m-\log _{2} w_{2}\right] w_{2} 2^{-m}+\ldots+x_{n}\left[m-\log _{2} w_{n}\right]\right. \\
& \left.w_{n} 2^{-m}\right)-\operatorname{round}\left(x_{1}\left[m-\log _{2} w_{1}\right] w_{1} 2^{p-m}+x_{2}\left[m-\log _{2} w_{2}\right] w_{2} 2^{p-m}+\ldots+(4)\right. \\
& \left.x_{n}\left[m-\log _{2} w_{n}\right] w_{n} 2^{p-m}\right)<2^{-(p+1)}
\end{aligned}
$$

According to (3) and (4), we can get $\mid x-\operatorname{round}\left(x_{1}\left[m-\log _{2} w_{1}\right] w_{1} 2^{p-m}+x_{2}[m-\right.$ $\left.\left.\log _{2} w_{2}\right] w_{2} 2^{p-m}+\ldots+x_{n}\left[m-\log _{2} w_{n}\right] w_{n} 2^{p-m}\right) \mid<2^{-(p+1)}+2^{-(p+1)}=2^{-p}$ that is to say $|x-x[p]|<2^{-p}$.

For example, when we want to compute $x=x_{1}+y_{1} * y_{2}+x_{3}+x_{4}+x_{5}$ that is $x=x_{1}+x_{2}+x_{3}+x_{4}+x_{5}$, where $x_{2}=y_{1} * y_{2}$ and $x_{1}, x_{2}, x_{3}, x_{4}, x_{5}$ are SCCs. We can appoint $x_{1}, x_{3}, x_{4}, x_{5}$ 's weight to be 1 and $x_{2}$ 's weight to be 4 . Then when we need to get $x[p]$, we should compute $x_{1}[p+4], x_{2}[p+2], x_{3}[p+4], x_{4}[p+4]$, $x_{5}[p+4]$ which is more efficient than compute $x_{1}[p+4], x_{2}[p+4], x_{3}[p+4]$, $x_{4}[p+4], x_{5}[p+4]$ that only use algorithm 3 to balance the precision. According to Algorithm 2 we can get $x_{2}[p+2]$, and then we use Algorithm 4 to compute the whole expression. 


\section{Conclusions and Discussions}

One of our aim is to avoid the unnecessary precision growth which has been talked in [17/18, in this paper we supply Algorithm 3 to balance every item in an expression including addition and subtraction. This way indeed improves exact arithmetic's speed. For example, we will do a computation that gets the sum of the harmonics series $\sum_{i=1}^{n} \frac{1}{i}$. The Table 1 below shows Algorithm 3's performance is much better than xrc1.1 which is an implementation based on V.Mnissier-Morain's Algorithm [3. The software xrc1.1 can be gotten from 21.

Table 1. Computing $\sum_{i=1}^{n} \frac{1}{i}$

\begin{tabular}{|c|c|c|c|}
\hline $\mathrm{n}$ & result'sprecision & $\mathrm{xrc1.1( \textrm {ms } )}$ & Algorithm 3 (ms) \\
\hline 1000 & 100 & 9.641 & 4.92 \\
\hline 1000 & 1000 & 77.377 & 10.948 \\
\hline 1000 & 10000 & 168.708 & 161.989 \\
\hline 5000 & 100 & 141.016 & 58.564 \\
\hline 5000 & 1000 & 187.183 & 106.209 \\
\hline 5000 & 10000 & 455.335 & 364.182 \\
\hline 10000 & 100 & 373.373 & 81.788 \\
\hline 10000 & 1000 & 446.627 & 150.112 \\
\hline 10000 & 10000 & 956.082 & 619.227 \\
\hline
\end{tabular}

Table 2. Computing $\sum_{i=1}^{n}\left(\frac{1}{i *(i+1)}+\frac{1}{i}\right)$

\begin{tabular}{|c|c|c|c|c|}
\hline $\mathrm{n}$ & result'sprecision & $\operatorname{xrc} 1.1(\mathrm{~ms})$ & Algorithm 3 (ms) & Algorithm $4(\mathrm{~ms})$ \\
\hline 100 & 100 & 3.358 & 4.909 & 2.901 \\
\hline 100 & 1000 & 11.811 & 10.911 & 10.256 \\
\hline 1000 & 100 & 162.803 & 81.511 & 65.246 \\
\hline 1000 & 1000 & 220.861 & 150.396 & 133.057 \\
\hline 10000 & 100 & 19166.903 & 324.03 & 288.016 \\
\hline 10000 & 1000 & 21070.736 & 900.51 & 894.279 \\
\hline
\end{tabular}

But our way can only solve some of this problem. For example, $x_{1} * x_{2} * x_{3}$ also should have a way to make sure that every item has the similar precision, but because the result of multiplication's interval is too complex we failed to get an easy means just like we compute addition or subtraction. In the future, we may find a way to completely solve this problem.

Another aim is to take into account the operation's complexity which is first introduced by us. We control every item's precision according to its complexity. In this way, we can improve the performance of the exact arithmetic furthermore. For example, we will do a computation that gets the value of $\sum_{i=1}^{n}\left(\frac{1}{i *(i+1)}+\frac{1}{i}\right)$. The Table 2 shows that Algorithm 4 has a better performance than xrc1.1 and Algorithm 3. 
There is also a question in this algorithm that how large the weight should be. In our implementation, we can easily appoint it by ourselves according to test many times basic operations and we also can adjust it according to need.

Acknowledgements. The research was supported by Chinese 973 Program (2004CB719400), and the National Science Foundation of China (60403047, 60533070 ). The second author was supported by the project sponsored by a Foundation for the Author of National Excellent Doctoral Dissertation of PR China (200342), and a Program for New Century Excellent Talents in University (NCET-04-0088).

\section{References}

1. A. Edalat, R. Heckmann, Computing with real numbers: (i) LFT approach to real computation, (ii) Domain-theoretic model of computational geometry, Lecture Notes in Computer Science, vol.2395, Springer, 2002, pp.193-267.

2. J. Blanck, Efficient exact computation of iterated maps, The Journal of Logic and Algebraic Programming, vol.64, 2005, pp. 41-59.

3. V. Mnissier-Morain, Arbitrary precision real arithmetic: design and algorithms. The Journal of Logic and Algebraic Programming, Vol.64, 2005, pp. 13-19.

4. W. Gosper, Continued fraction arithmetics, Technical Report HAKMEM Item 101B, Artificial Intelligence Memo 239, MIT, 1972.

5. Jen Vuillemin, Exact real computer arithmetic with continued fractions, IEEE Transactions on Computers, Vol. 39, 1990, pp. 1087-1105.

6. E.Bishop, D.Bridges, Constructive Analysis, Springer-Verlag, 1985.

7. Hans-J. Boehm, Robert Cartwright, Mark riggle, and Michael J.O'Donnell, Exact real arithmetic: A case study in higher order programming, In Proceedings of the 1986 Lisp and Functional Programming Conference, 1986, pp. 162-173.

8. V. Lee, Optimizing Programs over the Constructive Reals, PhD thesis, Rice University, 1991.

9. Hans-J. Boehm The constructive reals as a Java library, The Journal of Logic and Algebraic Programming, vol.64, 2005, pp. 3-11.

10. P. Gowland, D. Lester, The correctness of an implementation of exact arithmetic, in: Proceedings of the Fourth Conference on Real Numbers and Computers, 2000.

11. D. Lester, P. Gowland, Using PVS to validate the algorithms of an exact arithmetic, Theoretical Computer Science, Vol. 291, 2003, pp. 203-218.

12. K. Briggs, Implementing exact real arithmetic in python, $\mathrm{C}++$ and $\mathrm{C}$, Theoretical Computer Science, vol.351, 2006, pp. 74-81.

13. P. Gowland, D. Lester A survey of exact arithmetic implementations, Lecture Notes in Computer Science, vol.2064, Springer, 2001, pp. 30-47.

14. J. Blanck, Exact real arithmetic systems:Results of competition, Lecture Notes in Computer Science, vol.2064, Springer, 2001, pp.389.

15. A. Edalat, P. J. Potts, A new representation for exact real numbers, Electronic Notes in Theoretical Computer Science, Vol.6, 1997, pp. 119-132.

16. P.J. Potts, Exact real arithmetic using M?bius transformations, PhD thesis, Imperial College, 1999.

17. Branimir Lambov, RealLib:An Efficient Implementation of Exact Real Arithmetic, http://www.bric.dk/ barnie/RealLib/, 2006. 
18. Joris van der Hoeven, Computations with effective real numbers, Theoretical Computer Science, vol.351, 2006, pp. 52-60.

19. Norbert Th. Mller, The iRRAM: Exact Arithmetic in $\mathrm{C}++$. Lecture Notes in Computer Science, vol.2064, Springer, 2001, pp. 222-252.

20. K. Weihrauch, An Introduction to Computable Analysis, Springer, 2000.

21. K. Briggs, xrc homepage, http://keithbriggs.info/xrc.html, 2005. 